\title{
Patientenzufriedenheit im QMR-Kontext: Modell, Methode und Ergebnisse
}

Das Potenzial von Routinedaten für die Erfassung und das Management der medizinischen Behandlungsqualität ist heute unbestreitbar. Allerdings sind Patienten nicht nur Kunden sondern auch Mitproduzenten des Behandlungsergebnisses. Deshalb ist medizinisches Qualitätsmanagement ohne Berücksichtigung der Patientenwahrnehmung nicht sinnvoll. Die Integration der Patientenzufriedenheit in das Qualitätsmanagement mit Routinedaten (QMR) ist eine Konsequenz, und wir berichten die Entwicklung und Validierung einer Methode zur Integration der Patientenbeurteilung in das QMR.

\section{Methoden}

In die Kurzversion des Pickerfragebogens (PPE15) wurden mit Erlaubnis des Picker-Instituts 3 zusätzliche Fragen zur Beurteilung des Spitalaufenthalts an unterschiedlichen Positionen integriert. Es handelte sich um Fragen aus den Bereichen „Wiederkommen“, „Weiterempfehlen“ und „Qualität“, welche auf Basis einer systematischen Literaturanalyse ausgewählt bzw. entwickelt worden waren. Die 3 Fragen lauteten im Einzelnen:

1. Würden Sie für dieselbe Erkrankung wieder in unser Spital kommen?

2. Würden Sie einem Freund/einer Freundin unsere Klinik weiterempfehlen, wenn er/sie ähnliche Hilfe benötigen würde?

3. Wie würden Sie die Qualität der Behandlung, die Sie erhalten haben, beurteilen?

Die Beurteilung der Fragen erfolgte nach dem Mixed-Method-Verfahren in einem qualitativen und einem quantitativen Ansatz: (a) Bei 61 Patientinnen und Patienten mit unterschiedlichen Erkrankungen (maximum variation sampling) wurde die Bedeutung der Antworten auf die 3 Fragen mittels semistrukturierten Interviews nachuntersucht. (b) Bei 508 von 1146 ausgegebenen Fragebögen (Antwortrate $44 \%$ ) wurde die Korrelation der 3 Fragen und die Abhängigkeit von den sonstigen Bewertungen, die mit den weiteren 15 Fragen des Pickerbogens abgegeben wurden, analysiert (Faktorenanalyse).

\section{Ergebnis und Schlussfolgerung}

Zur Erfassung der integralen patientenseitigen Bewertung eines Spitalaufenthaltes eignen sich Fragen, die unter sehr unterschiedlichen Behandlungsbedingungen (z.B. Trauma, Onkologie, Rheumatologie, Kardiologie) verstanden und eindeutig interpretierbar sind. Dies trifft für die Frage (1) „Würden Sie für dieselbe Erkrankung wieder in unser Spital kommen?" nahezu ohne
Einschränkung zu. Die Antwort auf diese Frage wird je nach medizinischem und persönlichem Kontext von individuell unterschiedlichen Faktoren bestimmt: Die Faktorenanalyse der zusätzlichen Fragen ist geeignet, die für Patientengruppen wesentlichen Faktoren zu identifizieren. Grundsätzlich kann die gewählte Kernfrage in unterschiedliche Fragebögen integriert werden. In das QMR kann mit der Beantwortung dieser einen Frage die wichtige Patientendimension integriert werden.

Autorenerklärung: Die Autoren erklären, dass keine relevanten finanziellen Verbindungen in Bezug auf dieses Manuskript bestehen.
M. Heberer ${ }^{1}$
C. Depner ${ }^{1}$
A. Prengel 1
C. Abshagen 1
M. Bergman ${ }^{2}$

Qualitätsmanagement

Schlüsselwörter

Patientenzufriedenheit

Qualität

Keywords

hospital quality

Dospital performance

patient assessment
Institut

1 Institut für Chirurgische

Forschung und Spitalmanagement, Universitätsspital Basel

2Institut für Soziologie, Universität Basel

Bibliografie

Dol $10.1055 / \mathrm{s}-0029-1242681$ Dtsch Med Wochenschr 2009; 134: S327 - (c) Georg Thieme Verlag KG Stuttgart · New York . ISSN 0012-0472

Korrespondenz Prof. Dr. Michael Heberer Institut für Chirurgische Forschung und Spitalmanagement Universität Basel Spitalstr. 21 4031 Basel, Schweiz eMail mheberer@uhbs.ch 